\title{
Characteristics of Speed Line Cutter and Fringe Analysis of Workpiece Surface
}

\author{
Wang Shuai \\ Shenyang Aerospace University, Shenyang Liaoning 110136
}

\begin{abstract}
Easy to operate, speed line cutter has a high machining cost performance, so is very popular among the majority of users. The precision of guide rails, screws and nuts used in most of the machines is not high, and the machine control cannot compensate for the screw pitch error, clearance during the transmission and machining error due to electrode wear. Furthermore, control signal may also be lost in control process. The development of speed line cutter focuses on the quality and machining stability of CNC speed line cutter. This article makes an analysis about the impact of machine's inherent characteristics on machining workpiece surface, and concludes that analysis shall be made on the irregular fringe, therefore to heighten the machining precision.
\end{abstract}

Keywords: speed line; multiple cutting; precision; fringe

With the development of mold and die industry, the increasing proportion of precision mold manufacturing eagerly requires electrospark cutting machining to ensure its fast speed, fairly good surface machining quality and relatively high machining precision. The changes in space and shape for wire-electrode analyzed via machine equipment's precision realizes the overlapping of the front and rear cutting spacial locations, and makes reciprocating cutting fringe of Speed Line more obvious, therefore to achieve a fringe-free and multiple cutting, which improves the machining quality.

\section{Analysis on Improving Machining Precision of Line Cutter}

\section{1. $X$ and $Y$ Motion Straightness}

Straightness for one axis refers to the same in two planes. For example, the straightness for $\mathrm{X}$ axis means its straightness in $\mathrm{X}$ and $\mathrm{Y}$ plane, as well as in $\mathrm{X}$ and $\mathrm{Z}$ plane, just as a road that will not bend or fluctuate. The supporting plate of machine is supported by guide rail whose flatness, thus determines the motional straightness. Straightness loss may occur for two reasons: the first reason will be the flatness of guide rail itself, and the second shall be that of reference surface for guide rail installation. Only the integration of high-precision and stable guide rail, supporting plate and lathe bed shall make up the essential conditions for ensuring straightness. And the high-, low-temperature and aging treatments of guide rail, supporting plate and lathe bed are for the same purpose as above.

Inconsistency of rollers (steel balls) may cause reduced pressure points, or generally a seesaw phenomenon. Because the irregular motion of screws may also affect the guide rail. For instance, the unparalleled axial direction of screws with guide rail, inconsistent central height for screws and guide rail or, as a torsion may be applied between them or as the screws bend, will forcefully interfere in and disrupt the linear motion of guide rail during screws motional process. Mainly for that reason, we shall lay emphasis on precisely producing screws, nuts and their bases in a standard way.

Any dust or foreign matters, which will not only affect the flatness of guide rail motion, but also destruct and deform the guide rail, shall not be found in guide rail and rollaway nest no matter they are "V"- or "--"-shaped. It is required that no dust should be in guide rail, which is one of the regulations for machine maintenance, service and its long-lasting precision.

Verticality of the two axes is based on their respective straightness. As the error of straight line is reflected during verticality measurement, the result of accumulated values will make the verticality measurement lose its accuracy. Therefore, we shall first ensure the respective straightness, and then verticality. The verticality of two axes totally depends on that of two groups of guide rails on supporting plates. During assembly, one group of guide rails are to be fixed and adjusted on reference surface until the other group is vertical to that surface and fixed. Then we can make pin holes on them so as to fix the verticality for the two guide rails on middle supporting plates. Stable and valid operations in the assembly and measurement shall be pursued, as well as the precision improvement. The control of intermediate technic index is very important, as the assembly, repair or practice for a period will all decrease the accuracy. If original installation uses the allowed error as much as possible, it will be losing most 
of its accuracy. For example, if the precision standard of one machine is $0.02 \mathrm{~mm}$, the internally controlled precision for the first assembly shall be under $0.012 \mathrm{~mm}$, and combined with the straightness accuracy of guide rail itself, verticality of the two axes can be ensured.

Like the straightness, the working state of screws is also essential for verticality. Any external force applied to the locating surface of guide rail at a certain angle will result in an unusual change to it. So once the excessive verticality of $\mathrm{X}$ and $\mathrm{Y}$ axes is found, we shall carefully check whether it is caused by deformation or misplacement of guide rail, or the interference from the screws motion. If it results from the guiding effect of guide rail, we shall repeat the adaptation process by respectively loosing and fastening the screws and nuts in several positions, and the direction and values of excess will be generally stable. But if it is due to the interference of screws and nuts motion, the regularity of direction and values will be lost. For that, the pins on guild rails shall not be blindly loosened and removed, because any operations without thinking are of no use. Once the fixed pins are loosened, we shall repeat the entire process of first assembly and adjustment aforementioned.

Any measurement and adjustment shall be performed after the guide rail motion becomes stable. If kick and torsional pendulum occur, indicating the guide rails are dirty or have foreign matters, we must do the cleaning and lubricating job before adjusting, which shall be bore in our mind.

\subsection{Maximum Conicity for Taper Machine}

Taper machine's taper cutting incorporates $\mathrm{U}$ and $\mathrm{V}$, and links to $\mathrm{X}$ and $\mathrm{Y}$ axes, which constitutes coordinated movements on the upper and lower surfaces. U,V and $\mathrm{X}, \mathrm{Y}$ determine the two ends of those surfaces respectively, and the track points of workpiece on those two surfaces lie exactly on the connecting line of the two ends. All the above processes form the basic principle of taper cutting, and the swinging degree of upper end is to be determined by the travel of $\mathrm{U}$ and $\mathrm{V}$.

The ratio of UV's maximum swinging degree $b$ and the centerline spacing of upper and lower guide wheels determine the size of $t$ angle, and $t$ is the maximum slope for cutting. The similar figures formula from computing control system can exactly convert the size of upper and lower surface of workpiece to that of UV and XY surfaces, and the system has little precision loss. But it shall be noted that the V-shaped notch will be in a desirable state only if the molybdenum filament is in a vertical position. Once it swings and $b>0$, V-shaped notch will interfere in the motion of molybdenum filament. The error caused by interference will be very small when t angle is less than 1.50, but it'll be obvious when $\mathrm{t}$ angle lies between 1.5 30; When it reaches $30 \sim 60$, the error can directly pose a threat to machining precision and cutting effect, though normal cutting is still able to be operated. However, when exceeding 60, not only the precision will be lost seriously, but also it is hard to maintain the normal cutting, and even the molybdenum filament will be detached from the notch. So generally, a simply constructed taper machine formed by taper device will be additionally installed in the linear machine, and the maximum cutting conicity is normally limited to $\pm 60^{\circ}$, which is more than enough for machining task of regular draft angle. The cutting of larger conicity relies on a special taper machine that shall structurally solve the problem of guide wheel and following UV swinging. There's no interference from grooves caused by swinging, and the cutting conicity is accurate in principle. But the negative result is that during the process of solving the following swinging problem, the entire performance can be reduced; motion lag and return difference occurs, with motion fidelity and accuracy greatly decreased. Moreover, the daily application, and the universality, stability, convenience and flexibility of linear cutting may be affected, too. It is hard to take into account all the above matters.

\subsection{The Error of Coordinate and Displacement in Machine}

Single-axis straightness, $\mathrm{XY}$ verticality and system return difference is mainly responsible for occurrence of error.

Speed line cutter does not realize closed-loop control, so that the return difference of machine driven system has become an important mark of precision for the entire machine. The return difference mainly comes from the following 5 aspects.

(1) Gear clearance, basically caused by driver gear between stepper motor and screw.

(2) Connecting keys clearance affecting return difference, especially from the large gear on screw, shall not be ignored even if it is little. The influence of clearance in motor shaft keys involves not only the return difference, but also the production of noise.

(3) Clearance between screw and nut; after leaving factory, the axial transmission clearance of the lead screw pair is normally below $0.003 \mathrm{~mm}$, but the product with poor quality cannot be ensured to be in the above state.

(4) Clearance between screw and bearing; this clearance is to be removed by axial adjustment of bearing's inner and outer rings. However, if the bearing is of poor quality, it will lack rotary flexibility after removing clearance, and once the rotation speed increases, the clearance will appear again. So the bearing here shall not be ignored.

(5) The general inflexibility transmitted by torque is relatively poor, and it causes flexible deformation on weak positions that will induce motion lag and then produce clearance.

The above mentioned 5 aspects jointly produce system return difference. In the actual machining, even the simplest closed graphic shall at least go through a process of removing return difference. Thus, the actual machining precision is normally twice as the irremovable return difference. If system return difference is $0.006 \mathrm{~mm}$, then it will be possible for machining precision to be $0.012 \mathrm{~mm}$. Another main reason for accuracy loss of displacement is verticality of the two axes and straightness of each axis. The accuracy loss of displacement is called error, but this error is random, so that is hard to be calculated. 


\section{Multiple Cutting Process of Speed Line Cutter}

The basic reason for low speed cutter to achieve excellent machining precision and other technical indexes is that it adopts multiple cutting processes. Therefore, researching a multiple cutting machining process for speed line cutter is an important way to improve its machining quality. In the following is the introduction of how to use twice-cutting machining process in improving productivity, reducing surface roughness of workpiece, raising its geometrical accuracy and increasing the service life of workpiece.

(1) Analysis on once-cutting process of speed line cutter

Taking the male and female die of blanking die as an example, after finishing die machining using once-cutting process by speed line cutter, a method of reducing the pulse width parameter of high frequency power, cutting the number of power amplifier tubes and limiting the output current to a range of $0.8 \sim 1.2 \mathrm{~A}$ is generally adopted for the purpose of acquiring a better machining precision and surface quality for machined workpiece. At this moment, the machining speed is low. This cutting method ensuring the precision and surface quality for machined workpiece at the cost of reducing machining efficiency is fairly effective when machining a workpiece with height below $10 \mathrm{~mm}$. But for a workpiece with height more than $30 \mathrm{~mm}$, the emulsion is hard to reach the machining surface and has a poor cooling effect, plus the high temperature of electric arc between molybdenum filament and workpiece, and the vulnerability of workpiece surface to sintering, etc. will result in a low quality surface for machined workpiece. And a relatively rough hardened layer with roughness ranged Ra 1. 6 3. $2 \mu \mathrm{m}$ will also appear and is difficult to clean.

(2) Analysis on twice-cutting process of speed line cutter

The first problem encountered in twice-cutting on speed line cutter is how to program. For machining program of once-cutting process, if taking the size of male die as a standard, the female die program can be programmed according to conventional die fit clearance. But twice-cutting program does not comply to conventional programming. From so many process analysis and repeated experiments, and considering that the repetitive positioning precision during twice-cutting is $2 \mu \mathrm{m}$, then based on the discharging clearance in twice-cutting, we conclude: when using twice-cutting method of machining, the unilateral size of female die set by its program shall be $5 \mu \mathrm{m}$ less than that set by once-cutting program. For instance, if the required fit clearance of male and female die of blanking die is 0.01 $\mathrm{mm}$, then the cutting program for male die shall be programmed according to drawing size, and the one for female die shall be programmed in accordance with zero-to-zero clearance.

The first cutting during the second cutting aims at improving machining efficiency, so the pulse width of high frequency power and the number of power amplifier tubes may be increased within the process of the first cutting, and the machining current parameter may use $1.0 \sim 1.5 \mathrm{~A}$ to finish the first cutting at a relatively high speed and then return to the beginning of cutting.

The nature of the second cutting process, a low-corrosive process of discharging, is to improve machining precision and surface quality for workpiece, so the pulse width of high frequency power and the number of power amplifier tubes shall be reduced. It shall be cutting when tracking current have equalized the machine operating speed and no-load speed.

In the actual operation, the key step that must be performed before the second cutting is wire fastening. The vibration of wire electrode is mainly responsible for the cutting quality of speed line cutter, and the reasons for the vibration mainly include dynamic imbalance and radial beat of wire storage cylinder, axial and radial endplay of guide wheel, wire electrode tension, etc.. This article discusses the cutting method based on existing equipment, so it only makes an analysis on wire electrode without analyzing the vibration source from equipment factor. The wire electrode will age and be consumed due to high-speed moving in machining process and high temperature resulting from discharging in cutting process, which make its diameter and tension reduced, amplitude increased. Therefore, wire fastening is a crucial step in the second cutting. Finally, high precision and surface quality may be achieved by increasing the tension of molybdenum filament and reducing its vibration during cutting procedure.

After using twice-cutting process, the dimensional accuracy of machined workpiece has been improved, with its surface stains effectively removed and surface roughness reduced to provide a smooth and bur-free texture. Furthermore, thanks to the discharging and the effective cooling of emotion during the second cutting, the hardened layers of cutting surface have been increased, thus to greatly lengthen the dies'service life. When cutting a square hole of $100 \mathrm{~mm} \times 100 \mathrm{~mm}$ on a steel part with thickness of $25 \mathrm{~mm}$ by DK7740 speed line cutter, different cutting methods will provide varied process parameters and machining effect.

In all, it is completely possible to improve machining and producing efficiency and technic indexes as long as the program is correctly programmed, and parameters reasonably selected and operation well-performed.

\section{Fringe Analysis on Workpiece Surface}

\subsection{Reversal Fringe}

Determined by electric corrosion principle, discharge ionization produces high temperature, then the hydrocarbon in liquid is decomposed to produce a lot of carbon black that will plate the positive pole due to the effect of electrical field. This phenomenon is used to compensate for electrode in EDM machining. In the line cutting, part of the lines is taken out of gap by wires, and other part will always plate the workpiece surface. It features more inlets than outlets for wires. So that is the reason for appearance of black and white canine-shaped fringes. The adhesive capacity of plating is related to temperature changes between the main part of workpiece and discharge channel, as well as the electric 
field intensity between the poles. That is to say, the carbon black plating phenomenon is a by-product of electric corrosion. Fringe appears as long as machining exists. The carbon black plating thickness is normally $0.01 \sim 2 u m$, and it is difficult to be removed due to its existence among peaks and valleys in discharge pits. So it can be burnished only after surface is polished and pits removed. As long as not being washboard-shaped accompanying the cutting surface, the adhesive carbon black without any shapes is not needed to be specially treated, for the cutting efficiency, dimensional accuracy and finish of metallic matrix are exactly what we focus on. If we expect a better vision and lighter-colored fringes, we could proceed to the following steps: more dilute cooler, slightly older machine, reduced machining voltage, closer frequency conversion tracking, etc. To completely eliminate the fringes, that is to say, to remove all the fringes produced, means that the wires will not be reversed, and liquid in which no emulsified hydrocarbon is contained changed for pure water. But in this case, the main advantages our speed line cutting boasts will be lost. Currently, multiple cutting remains the most effective method of removing reversal fringes, which is to make allowance of $0.005 \sim 0.02 \mathrm{~mm}$ along the outline, and cut again after correcting the cutting track. The outline without allowance shall be cut again along previous track. Integrated with adjustment of pulse machining parameters, this repetitive cutting will completely remove the fringes and improve the finish by a level. The basic condition for repetitive cutting is that machine shall have enough repetitive positioning precision and operation repeatability.

\subsection{Washboard Fringe}

As molybdenum filament reverses for once, a concave and convex will appear on cutting surface, and then form a regular shape-washboard shape, which is generally called "washboard fringe”. If there is not only the black and white reversal fringe, but also difference in sizes, which shall be not allowable. We shall find the reasons in positions mentioned below:

(1) Wire loosened or obvious different wire tightness of two ends of wire cylinders will make wires in operation swing greatly and obvious flexible bending when reversing. Consequently, super feed and short stop feed phenomenon must appear.

(2) Rotation inflexibility and instability of guide wheel bearing will result in difference between forward and backward rotary resistance, or in axial endplay.

(3) An electric conductor or a guide wheel applies too much resistance to molybdenum filament, which results in serious discrepancies between forward and backward tensions of wires in working area.

(4) Guide wheel or wire frame results in improper working position of guide wheel, unsymmetric V-shaped surfaces and separating or crossing of two V-shaped extension lines.

(5) Uneven feed related to line reversal results in lead or lag, and form a stair shape, similar to washboard fringe, on the oblique line and arc.
In all, the principal reason for appearance of washboard fringe is that wires travel in different paths in their working area (area between two guide wheels is called working area), and the difference of those two paths form the concave-convex amplitude of washboard. The basic reason for appearance of washboard fringe is mechanical reason. Guide wheel, bearing, electric conductor and the wire operating track are the main causes. Lead or lag induced by uneven feed, of course, is also one of the causes.

There's another kind of washboard fringe that has its cycle reversed not according to molybdenum filament but the periodic change of $\mathrm{X}$ and $\mathrm{Y}$ screws. The cause for that is the stair or bearing in which screw push carriage to move doesn't operate in a stable manner and induces ending beat, which may also be caused by relatively large clearance that allows foreign matters invasion. In short, once it is proved that the cutting defect varies with the periodic change of screw, we shall find reasons there. The best way to confirm the cause is to cut an oblique line at $45^{\circ}$, then its cycle and the reason of inducing defects will be clear.

Poor finish is just one of the consequences brought by washboard fringe. It can also result in efficiency reduction, wire breaking due to frequent short or open circuit, and an instant super feed will cause serious short circuit, thus to stop machining process.

\section{Conclusion}

This article makes an analysis on the influence the machine characteristics have on machined workpiece surface, and on the method to improve the machining effect of fringe-free cutting and multiple cutting of speed line machine. The machining quality of large and intermediate part cannot be ensured at present. Using AC servomotor driver, full-closed loop control and sectional compensation technology can achieve backlash, screw pitch and wire diameter compensation, which can largely improve positioning and machining precision. This will be an important developing direction of speed line cutter.

\section{References}

[1] Yang Zhenbu, Zhang Jianrong. Discussion on Development of "Intermediate Line" Electrospark Line Cutting Machine[J]. Electromachining and Mold,2012

[2] Li Xiaobo, etc..Brief Summary of Electrospark Machining Machine Corrective Maintenance[J].Electromachining and Mold,2002

[3] Zhang Shiqing, etc..Electrospark Machining Technology[J].Chinese Technology Expo, 2012

[4] Liu Rong, etc..Twice-Cutting Machining Process of Speed Line Cutter[J].Electromachining and Mold, 2011

[5] Pan Chunrong, etc..Treatments and Techniques of Cutting Remaining Parts of the Workpiece During Precision Line Cutting Machining[J].Mold Technology, 2000

[6] Li Jun, etc..The Influence the Twice-Cutting Process of Speed Line Electrospark Has on Mold Manufacturing[J].Hua Zhang, 2012

[7] Kou Yuanzhe.Analysis And Research on Factors that Influence Technic Indexes of Electrospark Contour Machining[J].Die Technology, 2008 